\title{
Strassenverkehr: 0,5 Promille und Nulltoleranz für Drogen
}

Lucia Rabia, Rechtsdienst FMH

Auf den 1. Januar 2005 treten neue Vorschriften im Strassenverkehrsrecht in Kraft. Sie sind Teil eines Massnahmenpakets zur Erhöhung der Verkehrssicherheit. Wir stellen mit Genugtuung fest, dass wesentliche von der FMH im Vernehmlassungsverfahren eingebrachte Anliegen berücksichtigt wurden.

Die folgenden Änderungen sind hier von besonderem Interesse:

\section{0,5-Promille-Grenze für Alkohol}

Der Blutalkoholgrenzwert wird gesenkt: Als fahrunfähig gilt, wer eine Alkoholkonzentration von mindestens 0,5 Promille (bisher 0,8\%o) aufweist oder eine Alkoholmenge im Körper hat, die zu einer solchen Konzentration führt [1].

\section{Nulltoleranz für Drogen}

Mit dem neuen Art. 2, Abs. 2, der Verkehrsregelnverordnung [2] gilt Fahrunfähigkeit grundsätzlich als erwiesen, wenn im Blut des Fahrzeuglenkers eine der nachstehenden Substanzen nachgewiesen wird:
a. Tetrahydrocannabinol (Cannabis);
b. freies Morphin (Heroin/Morphin);
c. Kokain;
d. Amphetamin (Amphetamin);
e. Methamphetamin;
f. MDEA (Methylendioxyethylamphetamin) oder
g. MDMA (Methylendioxymethamphetamin).

Kann der Fahrzeuglenker jedoch nachweisen, dass er solche Substanzen gemäss ärztlicher Verschreibung einnimmt, gilt Fahrunfähigkeit nicht bereits beim Nachweis dieser Substanzen als erwiesen [3]. In diesem Falle ist durch weitere Abklärungen festzustellen, ob tatsächlich Fahrunfähigkeit vorliegt oder nicht. Bei Hinweis auf Fahrunfähigkeit wird eine Begutachtung durch Sachverständige veranlasst [4].

\section{Ärztliche Untersuchung, Art. 142a VZV}

Gemäss bisheriger Vorschrift (Art. 140 VZV) hatte der mit der Blutentnahme beauftragte Arzt den Verdächtigten zusätzlich auf die medizinisch feststellbaren Anzeichen von Angetrunkenheit zu untersuchen.

Neu hat der mit der Blutentnahme beauftragte Arzt die betroffene Person auf die medizinisch feststellbaren Anzeichen von Fahrunfähigkeit aufgrund von Alkohol-, Betäubungs- oder Arzneimittelkonsum nach Massgabe des neuen, detaillierteren Formulars (Anhang 9 VZV) zu untersuchen.

Ist jedoch aufgrund des Verhaltens der betroffenen Person anzunehmen, dass als Ursache der Fahrunfähigkeit einzig Alkohol in Frage kommt, so kann die zuständige Behörde den Arzt von dieser Untersuchungspflicht entbinden.

\section{Auswirkungen für Sie in der Praxis}

Wie bis anhin werden Sie Ihre Patienten auf mögliche Nebenwirkungen von verschriebenen Medikamenten aufmerksam machen und gegebenenfalls auf die Beeinträchtigung der Fahrfähigkeit hinweisen. An dieser Verantwortung und Aufklärungspflicht ändert sich nichts.

Wenn Sie jedoch Medikamente mit den obenerwähnten Substanzen verschreiben, ist es ratsam, den Patienten selbst dann auf mögliche Probleme bei Verkehrskontrollen hinzuweisen, wenn die Einnahme des Medikamentes gemäss ärztlicher Anordnung die Fahrfähigkeit effektiv nicht beeinträchtigt.

Der Patient weiss dann, dass er im Falle einer Blutprobe Schwierigkeiten bekommen kann und sich rechtfertigen muss. Die neue strenge Vorschrift geht nämlich davon aus, dass Fahrunfähigkeit vorliegt, sobald eine der aufgelisteten Substanzen überhaupt nachgewiesen wird unabhängig davon, wie gering deren Menge ist. Empfehlen Sie dem Patienten, das ärztliche Rezept (oder eine Kopie davon), auf welchem die Bezüge in der Apotheke vermerkt sind, stets mit sich zu führen, oder stellen Sie ihm ein entsprechendes ärztliches Attest - für den Fall der Fälle! - aus. 